BULL. AUSTRAL. MATH. SOC.

VOL. 21 (1980), 13-20.

\title{
INTEGRAL INEQUALITIES AND APPLICATIONS
}

\author{
K. NARSIMHA REDDY
}

In this paper some nonlinear analogues of Gronwall's integral inequality are established and an application to differential equations is given.

\section{1 .}

Differential and integral inequalities play a prominent role in the study of existence, uniqueness, boundedness, stability and other qualitative properties of solutions of differential and integral equations. The aim of this paper is to establish some nonlinear integral inequalities with applications under general circumstances, and at the same time unifying many earlier works (cf. [1], [2], [3], [6], [10] and [11]) which claim their origin from the following.

LEMMA 1.1 (Gronwall [7], BelIman [2], Reid [11]). Let $u(t)$ and $f(t)$ be nonnegative continuous functions on $J=[0, a), a>0$, for which the inequality

$$
u(t) \leq c+\int_{0}^{t} f(s) u(s) d s, \quad t \in J,
$$

holds, where $C \geq 0$ is a constant. Then

$$
u(t) \leq c \exp \left(\int_{0}^{t} f(s) d s\right) \text { for } a \imath z \quad t \in J .
$$

Received 19 June 1979. This paper is a part of the M. Phil dissertation of the author written under the supervision of Dr V. Sree Hari Rao, Department of Mathematics, Osmania University, Hyderabad, India, for whose able guidance and constant encouragement the author is very much indebted. 


\section{2.}

In this section we develop some auxiliary results concerning the existence of maximal solutions of initial value problems.

$B C(I)$ denotes the space of bounded continuous functions defined on $I=[0, \infty)$. It is easy to see that $B C(I)$ is a Banach space with the norm defined by

$$
\|x\|=\sup _{t \in I}|x(t)|
$$

Let $S_{\rho}$ be the set $\{x \in B C(I):\|x\| \leq \rho, \rho>0\}$. Under the following hypotheses we establish the existence of the maximal solution to the initial value problem

$$
\begin{aligned}
r^{\prime}(t)=f(t) & {[h(t)+Q(C(t)+r(t))} \\
& \left.\quad+\int_{0}^{t} g(s)\{h(s)+Q(C(s)+r(s))\}\right] d s, r(0)=r_{0} .
\end{aligned}
$$

THEOREM 2.1. Assume the following hypotheses:

(i) the functions $u, h, f, c, g: I \rightarrow I$ are continuous;

(ii) $Q: I \rightarrow I$ is nondecreasing continuous function; and

(iii) there is a positive number $\rho$ such that

$r_{0}+\int_{0}^{t} f(s)\left[h(s)+Q(C(s)+\rho)+\int_{0}^{s} g(\tau)\{h(\tau)+Q(C(\tau)+\rho)\} d \tau\right] d s \leq \rho, \quad t \in I$.

Then there exists the maximal solution to the initial value problem (2.1) in $S_{\rho}$.

The proof of this result may easily be constructed following the proof of Theorem 1 of [5].

3.

We state the following result.

THEOREM 3.1. If under the hypotheses (i) and (ii) of Theorem 2.1, the inequality 
(3.1) $u(t) \leq h(t)+Q\left(C(t)+\int_{0}^{t} f(s) u(s) d s+\int_{0}^{t} f(s)\left(\int_{0}^{s} g(\tau) u(\tau) d \tau\right) d s\right)$ holds, then for all $t \in I$ the inequality

$$
u(t) \leq h(t)+Q(C(t)+r(t))
$$

also holds, where $r(t)$ is the maximal solution of (2.1).

Proof. Let

$$
v(t)=C(t)+\int_{0}^{t} f(s) u(s) d s+\int_{0}^{t} f(s)\left(\int_{0}^{s} g(\tau) u(\tau) d \tau\right) d s,
$$

so that (3.1) becomes

$$
u(t) \leq h(t)+Q(v(t)) \text { and } v(0)=C(0) .
$$

Also let $m(t)=v(t)-C(t)$. Then $m(0)=0$ and using (ii) we have

$$
\begin{aligned}
& m^{\prime}(t) \leq f(t)\left[h(t)+Q(C(t)+m(t))+\int_{0}^{t} g(s)(h(s)+Q(C(s)+m(s))) d s\right], \\
& t \in I \text {. }
\end{aligned}
$$

In view of Theorem 2.1 and Theorem 1.4 .1 of [8] finally it follows that $m(t) \leq r(t)$ for all $t \in I$, where $r(t)$ is the maximal solution of (2.1). This completes the proof.

It may be observed that Theorem 3.1 improves and includes several classical results.

REMARK 3.1. To see this in what follows, choose $Q(y) \equiv y$, $C(t) \equiv 0$ in (3.1) of Theorem 3.1 and $p \equiv 1$ in (2.1) of Theorem 1 of [10]. Then we have the following inequality

$$
u(t) \leq h(t)+\int_{0}^{t} f(s) u(s) d s+\int_{0}^{t} f(s)\left(\int_{0}^{s} g(\tau) u(\tau) d \tau\right) d s, \quad t \in I .
$$

It is natural to expect the final estimates to agree as the basic inequalities in both cases are the same. Unfortunately the conclusion of Theorem 3.1 for the above choices is not in agreement with that of Theorem 1 of [10]. This eventually shows that the proof of Theorem 1 of [10] is incorrect and the correct estimate follows from Theorem 3.1 of this paper.

REMARK 3.2. For various choices of $Q$ we see that Theorem 3.1 
includes various inequalities of Gronwall-Bellman type which may be seen in [8].

THEOREM 3.2. Assume the following:

(i) $u(t), h(t), f(t)$ and $g(t)$ are nonnegative continuous functions defined on $I$;

(ii) $\Omega$ and $Q$ are nonnegative, nondecreasing, continuous fructions on $I$ and $\Omega(0)=0$.

If the inequality

$$
u(t) \leq h(t)+Q\left(C+\int_{0}^{t} f(s) u(s) d s+\int_{0}^{t} g(s) \Omega(u(s)) d s\right)
$$

holds for alz $t \in I$ and $C>0$, then

$$
u(t) \leq h(t)+Q(r(t))
$$

where $r(t)$ is the maximat solution of

$$
r^{\prime}(t)=f(t)(h(t)+Q(r(t)))+g(t) \Omega(h(t)+Q(r(t))), \quad r(0)=C .
$$

The proof of this theorem runs along similar lines to that of Theorem 3.1 .

REMARK 3.3. If we choose $\Omega \equiv 0, Q(y) \equiv y$, then Theorem 3.2 reduces to a generalized form of the Gronwall-Bellman inequality (cf. [8], Corollary 1.9.1). Also if $h \equiv 0, f \equiv 0$ and $Q(y) \equiv y$, we see that Theorem 2.3 of $[3]$ follows at once.

\section{4.}

It is found in most of the earlier works (cf. [1], [2], [6], [7] and [10]) that continuity conditions are placed on the functions involved, which seems to be rather too stringent as most processes in nature exhibit discontinuous phenomena (for example, automatic controls, heart beats, and so on). Therefore in this section efforts are made to relax the continuity conditions. The dropping of continuity conditions enables us not only to enlarge the class of functions but also to construct more natural and realistic mathematical models.

Now we establish an integral inequality of Bellman-Bihari type under more general conditions. First we state the following lemma. 
LEMMA 4.1. Suppose that $n(t)$ is a positive monotonic, nondecreasing, integrable function and $u, f: I \rightarrow I$ are integrable functions such that

$$
u(t) \leq n(t)+\int_{0}^{t} f(s) u(s) d s
$$

holds for $t \in I$. Then

$$
u(t) \leq n(t) \exp \left(\int_{0}^{t} f(s) d s\right) \text { for alz } t \in I .
$$

The proof of this lemma runs along similar lines to that of Lemma 3.1 $[2$, p. 58].

The main result of this section is the following:

THEOREM 4.1. Assume

(i) $g$ defined on $I$ is an integrable function which does not change its sign on $I$,

(ii) $u, f: I \rightarrow I$ are integrable on $I$, and

(iii) $\Omega$ is a monotonic, submultiplicative, integrable function on $I$ and $\Omega(0)=0$.

Further suppose that the inequality

$$
u(t) \leq K+\int_{0}^{t} f(s) u(s) d s+\int_{0}^{t} g(s) \Omega(u(s)) d s, \quad t \in I,
$$

holds, where $K>0$ is a constant.

If either $\Omega$ is nondecreasing and $g \geq 0$ or $\Omega$ is nonincreasing and $g \leq 0$ then the inequality

(4.2) $u(t) \leq \exp \left(\int_{0}^{t} f(s) d s\right) G^{-1}\left[G(K)+\int_{0}^{t} g(s) \Omega\left(\exp \left(\int_{0}^{s} f(\tau) d \tau\right)\right) d s\right]$ also holds, as long as $G(K)+\int_{0}^{t} g(s) \Omega\left(\exp \left(\int_{0}^{s} f(\tau) d \tau\right)\right) d s$ lies in the domain of definition of $G^{-1}$, the inverse of $G$, where 


$$
G(\alpha)=\int_{y_{0}}^{\alpha} \frac{d s}{\Omega(s)}, \quad 0<y_{0} \leq \alpha
$$

Proof. Set

$$
n(t)=K+\int_{0}^{t} g(s) \Omega(u(s)) d s, \quad t \in I,
$$

so that $n(0)=K>0$ and

$(4.4)$

$$
n^{\prime}(t)=g(t) \Omega(u(t)) \text { for almost all } t \text { in } I,
$$

and also

$$
u(t) \leq n(t)+\int_{0}^{t} f(s) u(s) d s
$$

It is easy to see that $n(t)$ satisfies the requirements of Lenma 4.1 . In view of (4.5), (iii) and Lemma 4.1, we obtain

$$
u(t) \leq n(t) \exp \left(\int_{0}^{t} f(s) d s\right)
$$

and further,

$$
\frac{g(t) \Omega(u(t))}{\Omega(n(t))} \leq g(t) \Omega\left(\exp \left(\int_{0}^{t} f(s) d s\right)\right) \text { for all } t \in I .
$$

Also from the definition of $G$ and $(4.4)$ we have

$$
\frac{d}{d t} G(n(t))=\frac{g(t) \Omega(u(t))}{\Omega(n(t))} \leq g(t) \Omega\left(\exp \left(\int_{0}^{t} f(s) d s\right)\right) \text { for } t \in I .
$$

Integrating between 0 and $t$, we get

$$
G(n(t)) \leq G(K)+\int_{0}^{t} g(s) \Omega\left(\exp \left(\int_{0}^{s} f(\tau) d \tau\right)\right) d s \text { for all } t \in I \text {. }
$$

This again implies that

$$
n(t) \leq G^{-1}\left[G(K)+\int_{0}^{t} g(s) \Omega\left(\exp \left(\int_{0}^{s} f(\tau) d \tau\right)\right) d s\right]
$$

as $G^{-1}$ is nondecreasing. The final result follows in view of (4.6). 
REMARKS 4.1. 1. Dhongade and Deo have studied this inequality under a more restricted hypotheses. For details one may refer to [6].

2. If $\Omega \equiv 0$ and $f$ is nonnegative and continuous, Theorem 4.1 reduces to the celebrated Gronwall-Bellman inequality.

3. The choice $f \equiv 0$ continuity in place of integrability reduces Theorem 4.1 to Lemma 1 of Beesack [1, pp. 81-83].

5.

As an application of our results we consider the following differential equation

$$
x^{\prime}=f(t, x)
$$

where $f$ is defined in a certain region $D$ of the $(t, x)$-plane, $\left(t_{0}, x_{0}\right) \in D$ and obtain a uniqueness result for the solutions of (5.1).

THEOREM 5.1. If the function $f(t, x)$ satisfies the condition that, for $\left(t, x_{1}\right),\left(t, x_{2}\right) \in D$,

$$
\left|f\left(t, x_{2}\right)-f\left(t, x_{1}\right)\right| \leq w\left(\left|x_{2}-x_{1}\right|\right)
$$

where $W(u)$ is integrable and nondecreasing for $u \geq 0, W(u)>0$ for $u>0$ and $w(0)=0$ and further if $\int_{0}^{u} \frac{d s}{W(s)}$ is divergent for $u>0$, then there exists at most one function $\varphi$ in $D$ such that $\varphi$ satisfies (5.1) almost everywhere on $D$ and $\varphi\left(t_{0}\right)=x_{0}$.

This theorem may be proved by suitably modifying the proof of the result of $[3, \S 4]$.

\section{References}

[1] P.R. Beesack, "On integral inequalities of Bihari type", Acta Math. Acad. Sci. Hungar. 28 (1976), 3i-88.

[2] Richard Bellman, Kenneth L. Cooke, Differential-difference equations (Mathematics in Science and Engineering, 6. Academic Press, New York, London, 1963). 
[3] I. Bihari, "A generalization of a lemma of Bellman and its application to uniqueness problems of differential equations", Acta Math. Acad. Sci. Hungar. 7 (1956), 81-94.

[4] Jagdish Chandra and B.A. Fleishman, "On a generalization of the Gronwall-Bellman lemma in partially ordered Banach spaces", $J$. Math. Anal. App I. 31 (1970), 668-681.

[5] J. Chandra and V. Lakshmikantham, "Some point-wise estimates for solutions of a class of nonlinear functional - integral inequalities", Ann. Mat. Pura Appl. (4) 94 (1972), 63-74.

[6] U.D. Dhongade and S.G. Deo, "Some generalizations of Bellman-Bihari integral inequalities", J. Math. Anal. App I. 44 (1973), 2l8-226.

[7] T.H. Gronwall, "Note on the derivatives with respect to a parameter of the solutions of a system of differential equations", Ann. of Math. (2) 20 (1918-1919), 292-296.

[8] V. Lakshmikantham and S. Leela, Differential and integral inequalities. Volume I: Theory and applications (Mathematics in Science and Engineering, 55. Academic Press, New York and London, 1969).

[9] K. Narsimha Reddy, "Existence and uniqueness problems of differential equations through inequalities" (M.Phil. dissertation, Osmania University, Hyderabad, 1979).

[10] B.G. Pachpatte, "Integral inequalities of Gronwall-Bellman type and their applications", J. Math. Phys. Sci. 8 (1974), 309-318.

[11] William T. Reid, "Properties of solutions of an infinite system of ordinary linear differential equations of the first order with auxiliary boundary conditions", Trans. Amer. Math. Soc. 32 (1930), 284-318.

Department of Mathematics,

Osmania University,

Hyderabad 500007 ,

India. 